\title{
La colonisation française de l'Illinois : une réévaluation
}

\section{Margaret Kimball Brown}

Volume 39, numéro 4, printemps 1986

URI : https://id.erudit.org/iderudit/304402ar

DOI : https://doi.org/10.7202/304402ar

Aller au sommaire du numéro

Éditeur(s)

Institut d'histoire de l'Amérique française

ISSN

0035-2357 (imprimé)

1492-1383 (numérique)

Découvrir la revue

Citer cette note

Brown, M. K. (1986). La colonisation française de l’lllinois : une réévaluation. Revue d'histoire de l'Amérique française, 39(4), 583-591.

https://doi.org/10.7202/304402ar d'utilisation que vous pouvez consulter en ligne.

https://apropos.erudit.org/fr/usagers/politique-dutilisation/ 
NOTE DE RECHERCHE

\section{LA COLONISATION FRANÇAISE DE L'ILLINOIS: UNE RÉÉVALUATION}

MARGARET KIMBALL BROWN

Prairie-du-Rocher, Illinois

L'expérience coloniale française au pays des Illinois n'a jamais fait l'objet d'une analyse systématique. Au début du siècle Clarence Alvord retrouva les archives notariales de la colonie et rédigea The Illinois Country, 1673-1818, sans doute la meilleure synthèse historique de l'Illinois avant que ce pays ne devienne un État. Sa connaissance des événements de l'ère coloniale et sa capacité de les situer dans le contexte plus large de la politique européenne et américaine étaient admirables. On peut cependant lui reprocher de sérieux défauts. C'était un érudit de la fin du 19e siècle qui possédait un sens de l'histoire, mais qui ignorait, comme d'ailleurs la plupart des savants de son époque, tout concept de culture dans le sens anthropologique du terme. Par ailleurs, sa conception idéalisée du système de valeurs des pionniers américains qu'il partageait avec nombre de ses contemporains et qui n'est pas encore complètement disparue, influença profondément son interprétation du Régime français. Dans son cas ces défauts sont particulièrement significatifs car, à cause de son expertise et de sa renommée, non seulement sa vision historique mais aussi ses préjugés et ses idées fausses concernant le fait français, transmis à des générations d'étudiants et d'historiens, façonnent toujours notre perception de la colonisation française de l'Illinois.

La préparation d'une étude socio-culturelle de Prairie-du-Rocher, l'un des derniers villages français de l'Illinois, m'a poussée à analyser ses origines, c'est-à-dire sa fondation sous le Régime français. Il fallut donc me débarrasser des idées reçues et des préjugés du $19 \mathrm{e}$ siècle transmis par l'histoire d'Alvord depuis plus de 75 ans, afin de décrire clairement l'évolution de cette communauté.

Le parti pris d'Alvord en faveur de la mentalité américaine par rapport à celle des colons français ne laisse aucun doute. Ses descriptions, toutefois, qui avaient pour but de déprécier les Français, nous inquiètent; à bien des égards les Français paraissent être des citoyens modèles! Des citations tirées de l'introduction de son volume sur Cahokia illustrent ce paradoxe.

Les Français ne purent rivaliser d'égoïsme avec les Américains et durent céder leur place... Ils étaient élevés dans le respect des lois 
et des magistrats... Les pionniers américains aimaient mieux faire justice eux-mêmes agissant comme juge, jury et exécuteur.

Dans leurs disputes, les Français ne voyaient aucun déshonneur à chercher une «réparation d'honneur» plutôt que d'avoir recours à des règlements de compte brutaux comme le faisaient les Américains.

Le meilleur exemple des différences [entre Français et Américains] se trouve dans leur façon de vivre. Le pionnier américain préférait une cabane isolée, sans décoration, sans meubles ni confort, et mal entretenue... Les Français, par contre, cherchaient le raffinement; ils avaient le goût de l'esthétique; ils étaient plus polis dans tous les sens du terme. Leurs petites maisons dans les villages, entourées de fleurs et de potagers qui étaient enfermés par des clôtures de pieux traduisaient des conceptions et une civilisation différentes.

Loin d'être perçus d'une manière négative dans ces citations, les pionniers américains incarnent les valeurs intellectuelles définies par Frederick Jackson Turner:

Une rudesse et une force, une perspicacité et une curiosité; une intelligence pratique et inventive, rapide à trouver des expédients; une emprise admirable des choses matérielles manquant de finesse mais capable d'atteindre de grands buts; une énergie inquiète et nerveuse; un individualisme à toute épreuve... 2

Voilà les valeurs qu'admirait Alvord et qui représentaient pour lui la «virilité anglo-saxonne».

Mais que veut-on mettre en relief ici? Avons-nous, à la fin du 18e siècle, deux communautés de colons qui s'affrontent? Est-ce une frontière? Des différences de «mentalité nationale» sont-elles responsables du contraste? A partir des documents d'époque nous pouvons reconstruire une image un peu plus réaliste des villages français. Qui étaient leurs habitants? D'où venaient-ils? Comment vivaient-ils? Quelle était leur mentalité.

Les premiers habitants français de l'Illinois étaient des voyageurs qui pratiquaient la traite des fourrures (ou plus exactement des peaux de chevreuil et de bison dans cette région). Les voyageurs étaient d'une nature indépendante et suscitaient de nombreuses plaintes quant à leur comportement. Ils ne respectaient pas les lois et ne s'établissaient pas sur des fermes. Ils étaient sûrement individualistes et «viriles». Passer sa vie à voyager en canot le long des rivières du Midwest américain n'était pas un travail pour les faibles. En général ils étaient analphabètes et manquaient de sens esthétique.

\footnotetext{
1 Clarence W. Alvord, Cahokia Records, 1778-1790, Vol. 2, (Virginia Series, No. 1, Collections of the Illinois State Historical Library, 1907), lxv.

2 Frederick Jackson Turner, The Frontier in American History (New York, Henry Holt \& Company, 1920), 37.
} 
Plus tard, des colons, parfois d'anciens voyageurs mariés à des femmes amérindiennes, arrivèrent. Ils furent suivis de militaires envoyés pour assurer l'ordre dans ces territoires, un ordre rendu nécessaire par le comportement des trafiquants de fourrures. Quelques voyageurs continuaient à y vivre en qualité de chasseurs et de marchands de fourrures pendant toute la période coloniale, mais à la périphérie des communautés villageoises.

Les immigrants de l'Illinois arrivèrent directement du Canada ou de la France. Ils y venaient essentiellement pour les mêmes raisons que les colons britanniques de la côte atlantique - la chance d'avoir une terre et l'espoir d'améliorer leur sort. Plusieurs étaient des artisans qui s'établissaient dans ou près des villages pour devenir des habitants. La définition exacte du terme habitant en ce qui concerne l'Illinois dépasse le cadre de cette note de recherche, mais il est évident que ce terme s'appliquait aux personnes ayant acquis une terre et s'y étant installées (dans un an et un jour de la concession comme l'exigeaient les contrats). Alvord ne comprit jamais les structures d'occupation du sol puisqu'elles ne correspondaient pas à celles du régime seigneurial supposément en vigueur aux Illinois. Cette méprise sur la nature véritable de l'habitant, confondue dans l'esprit d'Alvord avec le voyageur et le coureur de bois, est peut-être à l'origine de sa mauvaise perception de la réalité. La description suivante illustre bien cette confusion:

La majorité, connue sous la rubrique habitants, était issue des classes populaires de France. Ces habitants étaient analphabètes et ignorants; leur mode de vie sur la frontière, loin des contrôles de la société civilisée, n'avait pas contribué à améliorer leurs qualités mentales ni morales, mais avait développé celles qui convenaient le plus à leur mode de vie. ${ }^{3}$

Tout en substituant les voyageurs aux habitants, cette citation semble indiquer que les colons français possédaient les mêmes qualités que les pionniers américains! Si c'était le cas, Alvord ne les approuvait pas. Malheureusement la perpétuation de cette fausse image des habitants domine l'histoire illinoise. Toutefois, les documents donnent une autre image, celle d'un habitant moyen alphabétisé qui maîtrisait un métier. Quelques-uns habitaient le long des routes qui sillonnaient la région, mais la majorité vivait dans des villages, à l'opposé des Anglosaxons plus «viriles».

Malgré l'importance de Kaskasia comme centre de traite, l'économie des Illinois était fondée sur l'agriculture depuis le début du $18 \mathrm{e}$ siècle. Les denrées illinoises descendaient le Mississippi jusqu'en basse Louisiane et assuraient la subsistance de cette dernière colonie lorsque les navires n'arrivaient pas de France. La basse Louisiane dépendait de

3 Alvord, Cahokia Records, xvii. 
ces importations de denrées pour sa survie, l'Illinois suffisait à ses besoins alimentaires. Cette caractéristique semble avoir échappé à Alvord qui déprécie les méthodes agricoles françaises et la quantité minime d'exportations. Certains documents attestent, cependant, l'envoi à la Nouvelle-Orléans de 100000 livres de farine dans un seul convoi. Une telle production nécessitait de vastes champs cultivés et une organisation gouvernementale suffisante pour recueillir, emmagasiner et expédier la farine et les autres denrées. L'Illinois avait dépassé le stade d'une économie autarcique assez tôt au 18e siècle.

Comment se présentait alors cette phase «pionnière» dans l'histoire de l'Illinois français? Un survol rapide du développement des deux communautés sert à démontrer les différences entre un nouvel établissement et une communauté bien enracinée.

Kaskasia fut fondée en 1703 comme centre de traite et de production agricole. Avec l'arrivée de militaires, on construisit le fort de Chartres à 25 kilomètres en amont et, en 1721-1722, on concéda des terres à des colons autour du fort. Un recensement de 1726 illustre la croissance rapide du fort de Chartres: 126 personnes contre 185 à Kaskasia. Toutefois il existait d'autres différences au point de vue économique. Les habitants de Kaskasia avaient mis 2210 arpents de terre en valeur et possédaient un cheptel évalué à 273 bêtes à cornes. Ceux du fort de Chartres, par contre, exploitaient seulemet 589 arpents de terre et ne détenaient que 89 bêtes à corne. Le nombre restreint de maisons — 47 contre 74 à Kaskasia - et la taille des familles — deux enfants par couple à Kaskasia contre moins d'un enfant par couple en moyenne au Fort de Chartres - caractérisent le mieux la jeunesse de cette communauté. Le peu d'enfants indique l'arrivée récente de jeunes familles, formées peut-être après la concession d'une terre.

Ces colons devaient défricher leurs terres, clôturer leurs champs, construire leurs maisons, des granges et des dépendances. Les terres fertiles les récompensaient d'abondantes récoltes, mais les travaux agricoles laissaient peu de temps pour les loisirs. L'agriculture n'était pas cependant leur seule occupation car la plupart maîtrisaient un métier: comme, par exemple, couvreur, tailleur d'habits, maçon, forgeron, etc.

Lors d'un second recensement en 1732, on dénombra 1258 arpents de terre en valeur au fort de Chartres et un cheptel qui avait triplé pour égaler celui de Kaskasia. Les familles avaient augmenté pour atteindre 2,5 personnes en moyenne ${ }^{4}$. L'accroissement des essarts et du cheptel nous désabuse de l'image de l'habitant paresseux et sans ambition qui est si répandue. Un officier militaire britannique décrit ainsi les habitants de Prairie-du-Rocher: «Les habitants sont travailleurs et produisent des grains en abondance et toute sorte de bétail. ${ }^{5}$

\footnotetext{
4 Archives nationales (Paris), Archives des colonies, A1 464, 1726; A1 464, 1732.

5 Philip Pittman, Present State of the European Settlements on the Mississippi (Facsimile edition, University of Florida Press, 1973), 391.
} 
Lorsque les premiers Américains pénétrèrent dans le pays des Illinois dans les années 1760 , ce n'était déjà plus une région de colonisation, car les villages français constituaient des communautés bien établies. En 1778, les troupes virginiennes, sous le commandement de George Rodgers Clark, s'emparèrent du terrritoire au nom des ÉtatsUnis. La plupart des soldats correspondaient à l'image que donne Alvord du pionnier américain et étaient détestés par les Français pour leur appropriation du bétail et de la nourriture contre le paiement en papier monnaie sans valeur. L'absence du soutien logistique de la Virginie laissait peu de choix aux soldats. Les troupes contribuèrent ainsi à façonner une mauvaise perception de la nouvelle république; néanmoins, les pionniers américains étaient acceptés par les anciens habitants.

Les pionniers américains occupèrent des terres à l'écart des établissements français, construisirent des cabanes de bois et cultivèrent la terre pour affirmer leur tenure. Isolées et sans protection, durant les années 1780 et 1790, les Américains devaient se réfugier dans des enclos fortifiés à cause du danger d'attaques amérindiennnes. Dans ces circonstances, leurs domiciles demeuraient primitifs jusqu'à ce que le danger d'attaques amérindiennes se résorba.

Les villages français, par contre, étaient denses et défendus par une milice bien entraînée. Les habitants étaient déjà à la deuxième ou troisième génération et l'époque des maisons primitives, pauvres et sans meubles était révolue. Leurs maisons étaient solides, parfois de pierre à deux étages, avec des bardeaux, des planchers de madriers et des cheminées en pierre. Les intérieurs étaient souvent plâtrés et blanchis à la chaux. Les meubles n'avaient rien de rustique car plusieurs des fabricants se désignent dans les documents comme maîtres ébénistes. Autour de la maison, à l'intérieur des clôtures de pieux, se trouvaient plusieurs dépendances - étable, grange, poulailler, puits, four et pigeonnier. Grâce à une production agricole abondante, les habitants avaient suffisament de surplus pour se payer des produits importés. Les inventaires font état de verreries, de porcelaine, de gobelets d'argent, de tapis, de rideaux de fenêtres, de miroirs et d'autres objets de luxe. Ainsi, le niveau de vie dans les villages français à la fin du 18e siècle était relativement élevé. Les basses terres très fertiles produisaient facilement des surplus destinés à l'exportation; le nombre de bêtes à cornes et de cochons s'accrut. Même si leur mode de vie peut nous paraître difficile, les habitants disposaient de loisirs.

Tous les visiteurs commentèrent l'amour des Français pour les bals et danses. La perception de ces activités varie selon l'observateur, mais les historiens y ont généralement vu un exemple de la frivolité d'une population sans ambition. Les commentaires d'Américains habitant la région à la fin du $18 \mathrm{e}$ siècle jettent une lumière différente sur ces amu- 
sements: «Parfois j'assistais aux bals avec d'autres enfants, mais loin d'être frivoles, ces danses étaient des écoles de bonnes manières. On dansait surtout le menuet.» ${ }^{6}$ "Aucune salle de danse n'était conduite avec plus d'honnetêté; le décorum et les bonnes manières étaient garantis par l'opinion publique.» ${ }^{7}$ Les historiens qui n'assistèrent jamais à ces bals les perçurent, peut-être à cause d'un puritanisme victorien, comme une perte de temps. Ainsi, ils voyaient les Français comme des hédonistes indolents et sans ambition. On peut mettre en cause l'éthique protestante. Les Français n'avaient pas le droit de s'amuser, ils auraient dû travailler!

Voici peut-être l'origine du conflit entre les valeurs pionnières et celles des communautés mieux établies. Quelle devait-être leur ambition? Les Français avaient atteint la belle vie aux Illinois; les Américains qui venaient d'arriver cherchaient encore à l'atteindre. Toutefois, les valeurs pionnières visaient des buts moins terre à terre. Frederick Jackson Turner estimait que les «idéaux du Midwest trouvent leur origine dans la cabane de bois ronds au milieu de la forêt... [Le pionnier] voyait plus loin que la vie dure dans une cabane de bois ronds et imaginait la belle maison confortable de ses enfants qui auraient des objectifs plus élevés. ${ }^{8}$ Les Américains étaient-ils si différents ou ne s'agitil pas simplement d'une différence dans le stade de développement? Ils arrivèrent avec leurs familles, d'autres se sont mariés avec des Françaises, apprirent la langue et adoptèrent les pratiques culturelles des anciens habitants. Tous les pionniers n'étaient pas analphabètes; le testament de l'un d'eux fait état de plusieurs livres dont un volume de Shakespeare et le Paradise Lost de Milton. Les valeurs pionnières tant louées par Alvord, Turner et d'autres ne correspondent pas exactement à la réalité, mais comme les Américains sont devenus le groupe dominant de l'Illinois, leur histoire n'a pas souffert autant de ces mauvaises perceptions de la réalité.

Les Français étaient bien établis et prospères au milieu du $18 \mathrm{e}$ siècle. Pourquoi leur en vouloir? Ils avaient déjà atteint un certain niveau de vie et de succès économique; leurs ambitions avaient été réalisées. Dans des conditions normales ils auraient formulé d'autres objectifs et, en effet, il semble bien que l'extension de l'aire de colonisation était un de leurs buts. Cette volonté trouve une manifestation au nord de Fort de Chartres dans la région de la prairie de l'Apacois, plus tard appellée Belle-Fontaine. Toutefois, peu de temps après la concession de ces terres, les Français subirent des transformations profondes imposées par l'extérieur. Les pionniers américains y jouèrent un rôle mineur; des changements dans le gouvernement et l'absence de lois provoquèrent l'essentiel de ces transformations.

6 Henry M. Brackenridge, Recollections of Persons and Places in the West (Philadelphia, J. B. Lippincott \& Co., 1868), 25.

7 John Reynolds, My Own Times (Ann Arbor, University Microfilms, 1968), 38.

8 Turner, op. cit., 153. 
Alvord décrit les forces affectant les communautés françaises, mais déprécie leur impact car, pour lui, la domination américaine résultait du plus grand dynamisme des pionniers. Cependant, les Français durent faire face à tant de bouleversements que la persistance de quelques-uns constitue une preuve de la ténacité de la population et de sa force. On dut abandonner l'ambition d'étendre la zone de colonisation et se replier pour survivre. Néanmoins, l'image du colon français pliant l'échine sous la domination agressive des Américains est fausse. Tour à tour ce furent les colons qui entrèrent en contact avec les différentes autorités pour protester contre l'anarchie et réclamer des lois et une autorité civile efficace.

Le premier revers fut la cession de la rive gauche du Mississippi par le traité de Paris en 1763. Les Français dans l'Illinois n'avaient pas connu la défaite et le Fort de Chartres n'avait même pas été menacé. L'Illinois n'était qu'un pion dans les jeux diplomatiques européens. Les Français, contrairement aux pionniers américains, n'avaient jamais cherché à être séparés de leur mère patrie et ils demeuraient Français. Plutôt que de devenir des sujets britanniques par simple hasard géographique, plusieurs colons déménagèrent sur l'autre rive du Mississippi dans le territoire espagnol. Le village de Chartres fut complètement déserté et plusieurs habitants quittèrent les autres communautés.

La perte du personnnel administratif, à l'exception des capitaines de milice, laissait la région sans gouvernement. Qui plus est, l'Illinois ne faisait partie d'aucune colonie britannique mais était régie, sans beaucoup de succès, par des militaires. Les colons députèrent des représentants pour rencontrer le général Gage à New York, le quartier général des troupes anglaises, pour se plaindre des militaires et pour demander l'établissement d'un gouvernment civil. Il fallut attendre 1774, cependant, avant que l'Illinois ne soit incorporé dans la nouvelle Province de Québec. Les Britanniques quittaient déjà la région à cette époque et les dernières troupes partirent en 1776, déléguant l'administration du territoire à un Français de Kaskasia.

En 1778, George Rogers Clark annexa les villages à la Virginie et tout semblait rentrer dans l'ordre. Une administration civile et des tribunaux utilisant le droit français furent établis. La joie des habitants fut de courte durée car aussitôt surgirent des problèmes avec les soldats et le papier-monnaie sans valeur qui circulait. Lorsqu'un officier français, de la Balme, arriva afin de recruter des hommes pour attaquer Détroit, les habitants espéraient un retour de la domination française. Malgré l'échec de l'expédition, celle-ci réveilla le sentiment national. Les habitants s'adressèrent au gouverneur de la Virginie pour lui annoncer qu'ils n'accepteraient désormais que des troupes françaises dans leurs villages. Les troupes virginiennes furent retirées en 1783. Cependant il 
n'existait aucun gouvernement civil et le chaos régna jusqu'à l'arrivée du gouverneur St. Clair en 1790.

La mauvaise opinion d'Alvord sur les Français provient sans doute de son analyse de leurs tentatives de gouverner Kaskasia. Néanmoins, il reconnnaît que leur population diminua de $77 \%$ entre 1783 et $1790^{9}$, lorsque les personnages les plus influents de la communauté émigrèrent vers Sainte-Geneviève et Saint-Louis laissant quelques-uns se battre dans un combat inégal contre les immigrants américains. Malgré l'absence d'un tribunal et des lois, les Français tentaient toujours d'obtenir un gouvernement civil. Cahokia réussit à maintenir un gouvernement efficace pendant cette période malgré ces contraintes.

Theodore Pease donne une image plus favorable des efforts français en soulignant l'expérience de Cahokia ${ }^{10}$. Ici, un tribunal administra efficacement les affaires de la communauté. L'échec à Kaskasia ne démontre pas l'incapacité des Français à se gouverner, mais provient du fait que la communauté avait perdu la majorité de ses habitants français et était devenue un centre d'activités et d'intrigues américaines.

Pendant cette période d'anarchie, les Français poursuivaient leur lutte pour obtenir un gouvernement. En 1786, les habitants adressèrent une pétition au Congrès américain réclamant un gouvernement civil. Malgré l'échec de cette démarche, le gouvernement despotique de Kaskasia fut renversé cette même année. Pour administrer ce territoire un Français du petit village de Saint-Philippe près du fort de Chartres fut nommé juge et Jean-Baptiste Barbeau de Prairie-du-Rocher, lieutenant de comté.

L'établissement de tribunaux et d'un gouvernement inaugura une nouvelle période de stablilité suite à l'arrivée du gouverneur St. Clair en 1790. Les trois principaux villages des Illinois eurent des comportements différents devant ces événements, mais seul Kaskasia, au centre du conflit, devint désorganisé; Cahokia, loin du conflit persévéra. Prairie-du-Rocher, située entre les deux, subit des influences de Kaskasia, mais put se maintenir. Le caractère français de ce village persista et les résidents participèrent au gouvernement.

La recherche sur les origines de Prairie-du-Rocher démontrent que les valeurs proposées par les historiens et leurs préjugés sont sans fondement. Néanmoins la vision d'Alvord demeure le fondement de notre perception des colons français de l'Illinois. Encore récemment, une histoire très répandue dans les écoles caractérisait la population française de «conservatrice, paresseuse et analphabète», citant un commen-

\footnotetext{
9 Clarence W. Alvord, The Illinois Country, 1673-1818 (Reprint, Chicago, Loyola University, The American West Series, 1965), 373.

Theodore Pease and Marguerite Pease, The Story of Illinois (1965).
} 
tateur qui affirma qu' «une existence aisée et des amusements festives constituaient leur principale ambition.» ${ }^{11}$ Ainsi se perpétuent les mythes qui influencent encore les gens de notre époque.

Pourtant les colons français n'étaient pas des paresseux, sans souci et incapables de se gouverner. Ils n'étaient pas ignorants, illettrés, ni immoraux. Ils sont restés alphabétisés, ont maintenu un haut niveau de vie, un ferme attachement à l'Église et ses préceptes, une ferme conviction dans les bienfaits de la loi et de l'ordre. Il est plus que temps de rejeter les anciens historiens et de développer une analyse cohérente de l'expérience coloniale française comme partie intégrante de l'histoire de l'Illinois.

Traduction: JOHN A. DICKINSON

11 Robert Howard, Illinois: A History of the Prairie State (Grand Rapids, William B. Ederman Publishing Co., 1972), 41. 